\title{
Conditions that may affect the results of susceptibility testing of Mycobacterium tuberculosis to pyrazinamide
}

\author{
YING ZHANG, SALLIE PERMAR and ZHONGHE SUN
}

Department of Molecular Microbiology and Immunology, School of Hygiene and Public Health, Johns Hopkins University, Baltimore, MD 21205, USA

\begin{abstract}
Pyrazinamide (PZA) is an important front-line anti-tuberculosis drug that is active only at acid pH. However, acid pH causes significant difficulty for PZA susceptibility testing. A common problem in PZA testing is false resistance caused by large bacterial inocula. This study investigated the relationship of false resistance to numbers of bacilli, $\mathrm{pH}$ and other factors that potentially affect susceptibility to PZA. Large inocula (10 ${ }^{7-8}$ bacilli/ml) of $M$. tuberculosis H37Ra caused significant increase in medium pH from 5.5 towards neutrality, and thus produced false resistance results. The increase in medium pH was determined to be a function of live bacilli; heat-killed bacilli had little or no effect. Susceptibility to PZA and its active derivative pyrazinoic acid (POA) was comparable on 7 H11 agar medium, but POA was less active than PZA in liquid medium containing bovine serum albumin (BSA), suggesting that susceptibility to PZA or POA was reduced in the presence of $\mathrm{BSA}$, because of its neutralising effect on medium $\mathrm{pH}$ and significant POA binding. A 3-month-old H37Ra culture was shown to be more susceptible to PZA exposure than a 4-day log-phase culture, suggesting that PZA is more active for non-growing bacilli. Finally, reserpine, an inhibitor of POA efflux pump, increased susceptibility to PZA even near neutral pH 6.8, with an MIC of $400 \mathrm{mg} / \mathrm{L}$ compared with $1000 \mathrm{mg} / \mathrm{L}$ without reserpine. These findings should have implications for understanding the mode of action of PZA and for PZA susceptibility testing.
\end{abstract}

\section{Introduction}

Pyrazinamide (PZA) is an important front-line antituberculosis (TB) drug. PZA plays a unique role in shortening the TB therapy from the previous 9-12 months to 6 months because it kills a population of semi-dormant bacilli that are not killed by other TB drugs [1]. PZA, an analogue of nicotinamide, is a prodrug that must be activated by bacterial pyrazinamidase/nicotinamidase to the active derivative pyrazinoic acid (POA), which kills Mycobacterium tuberculosis $[2,3]$. An earlier study identified the pncA gene encoding pyrazinamidase/nicotinamidase from $M$. tuberculosis and showed that mutation in pncA causes PZA resistance [3]. Subsequent studies have shown that mutation in pncA is the major mechanism of PZA resistance in $M$. tuberculosis [4-11]. The activity of PZA correlates with the acidity of the medium, being

Received 14 May 2001; revised version accepted 4 July 2001.

Corresponding author: Dr Y. Zhang (e-mail: yzhang@ jhsph.edu). most active at $\mathrm{pH} 5.5$, less active at $\mathrm{pH} 6$ and almost inactive at neutral $\mathrm{pH}[12-14]$. It has been shown that acid $\mathrm{pH}$ enhances the uptake and accumulation of POA in M. tuberculosis, which has been shown to have a defective efflux mechanism for POA [15]. While the target of POA is thought to be fatty acid synthesis pathway 1 [16], POA as a weak acid could potentially kill tubercle bacilli through recycling of POA leading to de-energised membrane as a possible mechanism of action [17].

PZA susceptibility testing is difficult and often unreliable because of the acid $\mathrm{pH}$ requirement for drug activity [18]. For that reason, many clinical microbiology laboratories do not perform PZA susceptibility testing and most drug-resistance surveys do not have PZA resistance data. One problem of PZA susceptibility testing is the inhibition by acid such that at least $10 \%$ of clinical isolates cannot be tested because they fail to grow on acid $\mathrm{pH}$ medium $(\mathrm{pH}$ 5.5) [19]. By far the most common problem is false resistance $[12,18]$, whereby a large inoculum of a sensitive strain can lead to false resistant results. The large inoculum is often 
difficult to control due to significant clumping of the bacilli. The use of the BACTEC TB 460 system has improved the reliability of PZA susceptibility testing [19]; however, problems of PZA testing are not completely eliminated and PZA testing remains the most difficult among all antimycobacterial drug testing. Furthermore, there have been inconsistent reports of susceptibility to POA in M. tuberculosis, and in many instances the POA MIC is often reported to be higher than the PZA MIC [20-22]. In one study, the POA MIC (240-480 mg/L) for M. tuberculosis was reported to be much higher than the PZA MIC $(16-50 \mathrm{mg} / \mathrm{L})$ [22]. However, a study from another group reported POA MIC values for M. tuberculosis in the range 16$32 \mathrm{mg} / \mathrm{L}$ in 7H10 liquid medium [23], which was comparable to the PZA MIC. This study was undertaken to determine the effect of various factors on susceptibility to PZA and POA.

\section{Materials and methods}

\section{Mycobacterial growth conditions and $p H$ measurements}

M. tuberculosis strain H37Ra was grown in 7H9 liquid medium (Difco) supplemented with Tween $80 \quad 0.05 \%$ and $\mathrm{ADC} 10 \%$ enrichment containing bovine serum albumin (BSA)-dextrose-catalase $0.5 \%$ (Difco) at $37^{\circ} \mathrm{C}$ for 2 weeks with occasional agitation. For determining the effect of inoculum size on $\mathrm{pH}$ changes, 3-week-old H37Ra cultures were concentrated five-fold by centrifugation and resuspension in $7 \mathrm{H} 9$ medium. The bacterial cell suspensions were counted with a haemocytometer and serial 10-fold dilutions were prepared to obtain an inoculum range $10^{3}-10^{8} / \mathrm{ml}$ contained in a volume of $5 \mathrm{ml}$ of $7 \mathrm{H} 9$ medium adjusted to either $\mathrm{pH} 5.5$ or $\mathrm{pH} 7.0$. At $3 \mathrm{~h}, 1,2,3,6$ and 8 days, $0.8-\mathrm{ml}$ portions were removed and the $\mathrm{pH}$ was measured with a pH meter (Corning). Heat-killed bacilli were prepared in a similar manner and used in the $\mathrm{pH}$ measurement; media without bacilli served as a negative control. The effects of BSA or fetal bovine serum (FBS) (Sigma) on medium $\mathrm{pH}$ were determined in simple salt Sauton's medium [24] at $\mathrm{pH} 5.5$ or 7.0. The $\mathrm{pH}$ of the medium was determined similarly to the method described above, with the exception that the $\mathrm{pH}$ measurement was performed $1 \mathrm{~h}$ after addition of BSA.

\section{Drugs and chemicals}

PZA and POA were purchased from Sigma and Aldrich Chemical Co. (St Louis, MO, USA), respectively. PZA was dissolved in distilled water at a concentration of $10 \mathrm{mg} / \mathrm{ml}$ and filter-sterilised. POA was dissolved in DMSO at a concentration of $100 \mathrm{mg} / \mathrm{ml}$. In addition, POA, which was not completely dissolved in water at $10 \mathrm{mg} / \mathrm{ml}$ and contained undissolved crystals at room temperature, was dissolved in $1 \mathrm{~N} \mathrm{KOH}$, forming potassium POA salt. Reserpine and valinomycin were purchased from Sigma and were dissolved in DMSO and ethanol at $1 \mathrm{mM}$ and $0.1 \mathrm{mM}$, respectively.

\section{PZA and POA susceptibility testing}

PZA and POA susceptibility testing for M. tuberculosis H37Ra was performed on acidic 7H11 or Sauton's agar plates (pH 5.6) as described previously [25]. Reserpine at 20,40,60 and $100 \mu \mathrm{M}$ and valinomycin at $0.2,0.4$, 0.6 and $1 \mu \mathrm{M}$, were incorporated into the $7 \mathrm{H} 11$ agar medium ( $\mathrm{pH} 5.5$ and 6.8) to determine the effect of these agents on PZA or POA susceptibility. To determine the effect of BSA on PZA or POA susceptibility in liquid medium, the experiment was performed in Sauton's liquid medium ( $\mathrm{pH}$ 5.6) containing BSA $0.25,0.5,1$ and $2 \%$ in duplicate. Each microtitration plate well contained $200 \mu \mathrm{l}$ of Sauton's medium with or without various concentrations of BSA and either PZA or POA $(60-240 \mathrm{mg} / \mathrm{L})$ and inoculated with $10^{6}$ bacilli $/ \mathrm{ml}$. Inoculated microtitration plates were then incubated without shaking at $37^{\circ} \mathrm{C}$ for 6 days when the plate was developed with 3-[4,5-dimethylthiazol-2-yl]2,5-diphenyltetrazolium bromide (MTT; Sigma) and the plates were read at $\mathrm{OD}_{570}$ in an ELISA reader as described previously [26]. The degree of growth inhibition by PZA or POA in the presence of BSA was expressed as 50\% inhibition over the control. This was because PZA or POA does not completely kill tubercle bacilli (only c. $50-70 \%$ ) in vitro at acid $\mathrm{pH}$ [1]. The above test was performed in 96-well microtitration plates for the convenience of this particular experiment and does not represent endorsement of the use of microtitration plates and MTT for PZA susceptibility testing.

\section{Survival of mycobacterial cultures of different ages upon exposure to PZA}

Log-phase cultures of 4-12-day-old and 2-3-monthold stationary phase cultures of M. tuberculosis H37Ra were grown in Sauton's medium as standing cultures. These cultures were centrifuged and washed with PBS buffer once. The bacterial cells were resuspended in an acidic phosphate-citrate buffer (disodium phosphate $2.5 \mathrm{~g}$, monopotassium phosphate $1 \mathrm{~g}$ and sodium citrate $0.1 \mathrm{~g}$ in $1 \mathrm{~L}$ of distilled water, $\mathrm{pH} 5.6$ ) and adjusted to the same cell density of $c$. $\mathrm{OD}_{600}=2$. The numbers of $\mathrm{cfu}$ in the cell suspensions were determined on $7 \mathrm{H} 11$ plates, and both bacterial suspensions gave comparable cfu counts. PZA was added to cell suspensions prepared from the $\log$ phase and the stationary phase cultures at $16,31,62,125,250,500$ and $1000 \mathrm{mg} / \mathrm{L}$ in $200 \mu \mathrm{l}$ of phosphate-citrate buffer, and the mixtures were incubated at $37^{\circ} \mathrm{C}$ for 3 days without shaking. The cell suspensions were then washed with PBS to remove PZA, and the degree of bacterial survival upon exposure to PZA was assessed by plating samples of cell suspensions on $7 \mathrm{H} 11$ plates, which were then incubated at $37^{\circ} \mathrm{C}$ for c. 3 weeks. The viability of the bacterial cells was also determined by MTT assay [26] as 
follows. After PZA exposure at $37^{\circ} \mathrm{C}$ for 3 days without shaking, the viability of the cells was assessed by subculture into fresh Sauton's medium in a 96-well microtitration plate and incubated at $37^{\circ} \mathrm{C}$ for 5 more days. The degree of killing by PZA exposure was expressed as percentage of inhibition over control without PZA.

\section{POA binding by $B S A$}

BSA was diluted in distilled water to $20,4,0.8$ and $0.16 \%$, then $100 \mu \mathrm{l}$ of each dilution were mixed with $100 \mu \mathrm{l}$ of $\left[{ }^{14} \mathrm{C}\right] \mathrm{POA}$ or $\left[{ }^{14} \mathrm{C}\right] \mathrm{PZA}(0.2 \mu \mathrm{Ci} / \mathrm{ml})$ in water and incubated at room temperature for $1.5 \mathrm{~h}$. $\left[{ }^{14} \mathrm{C}\right] \mathrm{POA}$ was prepared by hydrolysis of $\left[{ }^{14} \mathrm{C}\right] \mathrm{PZA}$ with $1 \mathrm{~N} \mathrm{KOH}$ for $2 \mathrm{~h}$ followed by neutralisation with $1 \mathrm{~N} \mathrm{HCl}$ and complete conversion was confirmed by thin-layer chromatography as described previously [15]. After incubation of BSA with either $\left[{ }^{14} \mathrm{C}\right] \mathrm{POA}$ or $\left[{ }^{14} \mathrm{C}\right] \mathrm{PZA}, 100$ $\mu 1$ of this mixture was loaded on Vivaspin tubes (Sartorius) with a mol. wt cut-off of $30 \mathrm{kDa}$, and centrifuged at $10000 \mathrm{rpm}$ in a microfuge for $5 \mathrm{~min}$. The unbound $\left[{ }^{14} \mathrm{C}\right] \mathrm{POA}$ was collected as a filtrate in an Eppendorf tube and activity was counted as described previously [15]. A significant decrease in radioactive $\left[{ }^{14} \mathrm{C}\right] \mathrm{POA}$ in the filtrate would indicate binding of POA by BSA, which would be trapped by the membrane in the Vivaspin tubes.

\section{Results}

\section{Effect of inoculum size on medium $p H$}

The effect of various inoculum sizes of H37Ra $\left(10^{3}-10^{8}\right.$ cells $\left./ \mathrm{ml}\right)$ on the medium $\mathrm{pH}(5.5)$ was tested over a period of 8 days. Higher inoculum sizes $\left(10^{7}\right.$ and $10^{8}$ cells $/ \mathrm{ml}$ ) caused an increase in $\mathrm{pH}$ over time towards neutral pH (Fig. 1). At day 8, an increase of about 1 unit and 1.5 unit in $\mathrm{pH}$ was seen with $10^{7}$ and $10^{8}$ cells $/ \mathrm{ml}$, respectively, whereas a small increase of $<0.3$ unit was seen with $10^{6}$ cells $/ \mathrm{ml}$, an inoculum commonly used in PZA susceptibility testing (Fig. 1a). The increased $\mathrm{pH}$ by large inocula was dependent on live cells (Fig. 1a), but not heat-killed cells $\left(10^{8}\right.$ cells $/ \mathrm{ml}$ ), which caused little or no increase in $\mathrm{pH}$ $(<0.3$ unit) (Fig. 1b).

\section{Relationship between $p H$ and PZA MIC}

The increase in medium $\mathrm{pH}$ by a large bacterial inoculum is expected to cause an elevated PZA MIC. Given that PZA is completely converted to POA, the relationship between medium $\mathrm{pH}$ and PZA MIC can be expressed in the form of the Henderson-Hasselbach equation, where $\mathrm{pH}=\mathrm{pKa}+\log \left[\mathrm{A}^{-}\right] / \mathrm{HA}$. The theoretical calculations based on this equation for the relationship between $\mathrm{pH}$ and PZA MIC are shown in Table 1. For example, for a medium $\mathrm{pH}$ of 5.5 , with the POA pKa of 2.9 [26], the anion $\mathrm{POA}^{-}$is 398 -fold more than the uncharged protonated HPOA $\left(\log \left[\mathrm{POA}^{-}\right] /\right.$
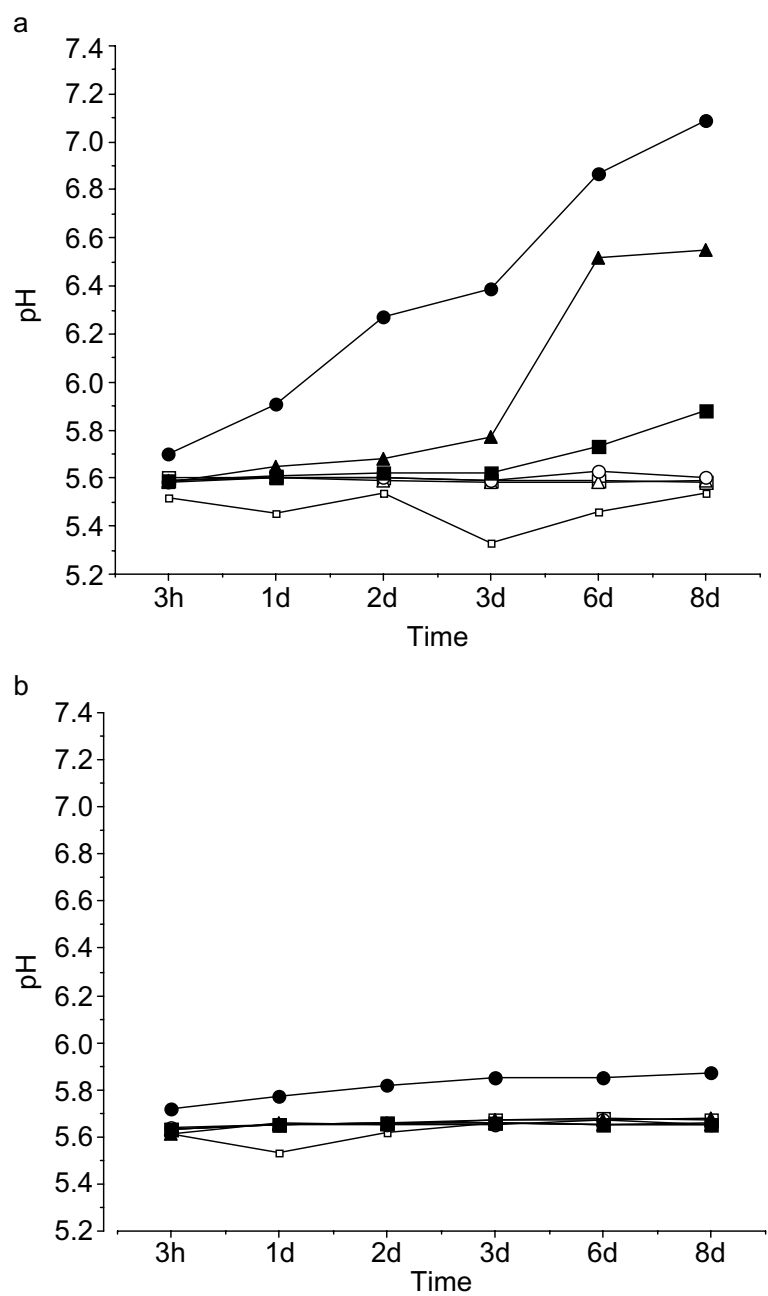

Fig. 1. Effect of inoculum size on acid medium $\mathrm{pH}$. $M$. tuberculosis H37Ra inocula of varying sizes which contained live bacilli (a) or heat-killed bacilli (b) were inoculated into 7H9 liquid medium at $\mathrm{pH} 5.6$ and medium $\mathrm{pH}$ was measured at different time points.

HPOA $=2.6$ ), whereas at medium $\mathrm{pH}$ of 6.0 , the anion $\mathrm{POA}^{-}$is 1259 -fold more than the uncharged HPOA $\left(\log \left[\mathrm{POA}^{-}\right] / \mathrm{HPOA}=3.1\right)$. Because it is the uncharged POA that gets into the cells and is thus the active species involved in killing, the POA MIC at $\mathrm{pH} 6.0$ is expected to be 3.2-fold higher than that at $\mathrm{pH} 5.5$. Therefore, based on PZA MIC at $\mathrm{pH} 5.5$ being $50 \mathrm{mg} / \mathrm{L}$, the PZA MIC at pH 5.8 and 6.1 should be 100 and $200 \mathrm{mg} / \mathrm{L}$, respectively. This prediction from the Henderson-Hasselbach equation fits well with results of a previous study showing the relationship between $\mathrm{pH}$ and PZA MIC, where at $\mathrm{pH}$ 5.5, 5.8 and 6.0, PZA MIC was found to be 50,100 and $200 \mathrm{mg} / \mathrm{L}$, respectively [14].

\section{POA versus PZA susceptibility}

To address the discrepancy between susceptibility to POA and PZA [21, 22], this study determined the POA MIC and PZA MIC for M. tuberculosis strain H37Ra on 7H11 agar at pH 5.5. POA is a weak acid that is poorly soluble in water [27], but could be dissolved in DMSO up to $100 \mathrm{mg} / \mathrm{ml}$ or in dilute $\mathrm{KOH}$ as a POA 
Table 1. Relationship between medium $\mathrm{pH}$, percentage of uncharged POA and PZA MIC

\begin{tabular}{lcccc}
\hline Medium $\mathrm{pH}$ & $\mathrm{POA}^{-} / \mathrm{HPOA}$ & HPOA $(\%)$ & $\begin{array}{c}\text { Theoretical PZA } \\
\text { MIC }(\mathrm{mg} / \mathrm{L})\end{array}$ & $\begin{array}{c}\text { Actual PZA MIC } \\
(\mathrm{mg} / \mathrm{L})\end{array}$ \\
\hline 4.5 & 39.8 & 2.5 & 5 & $\mathrm{NT}$ \\
4.8 & 79.4 & 1.26 & 10 & $\mathrm{NT}$ \\
5.0 & 125.9 & 0.8 & 16 & $\mathrm{NT}$ \\
5.5 & 398.1 & 0.25 & 50 & 50 \\
5.8 & 794.3 & 0.13 & 100 & 100 \\
6.0 & 1258.9 & 0.08 & 156 & 200 \\
6.8 & 7943.3 & 0.013 & 992 & 1000 \\
7.0 & 12589.3 & 0.008 & 1563 & $>1000$ \\
\hline
\end{tabular}

Values of $\mathrm{POA}^{-} / \mathrm{HPOA}$, \% HPOA were calculated by the Henderson-Hasselbach equation based on various medium $\mathrm{pH}$ values and $\mathrm{pKa}$ of POA being 2.9. The theoretical PZA MICs were values calculated with the same equation based on PZA MIC at $\mathrm{pH} 5.5$ being $50 \mathrm{mg} / \mathrm{L}$. The data on the actual PZA MIC at pH 5.5, 5.8 and 6.0 were from ref. 20, and PZA MIC at pH 6.8 was determined in this study. NT, not tested.

salt. Under these conditions, POA was completely dissolved and the POA MIC for M. tuberculosis H37Ra was found to be $32 \mathrm{mg} / \mathrm{L}$, which is no different from the PZA MIC.

\section{Effect of BSA on POA and PZA susceptibility}

On Sauton's agar medium, the POA MIC was $25 \mathrm{mg} / \mathrm{L}$, which is about two-fold lower than that on 7H11 agar medium $(\mathrm{MIC}=50 \mathrm{mg} / \mathrm{L})$. A major difference between Sauton's and 7H11 agar is that the latter contains BSA $0.5 \%$. When Sauton's agar medium was supplemented with BSA $0.5 \%$ (the same concentration as in $7 \mathrm{H} 11$ agar), the POA MIC was comparable to that on $7 \mathrm{H} 11$ agar $(50 \mathrm{mg} / \mathrm{L})$. These results suggest that BSA could decrease the susceptibility to POA. To address the effect of BSA on POA and PZA susceptibility more systematically, varying concentrations of BSA were tested in Sauton's liquid medium $(\mathrm{pH} \mathrm{5.5),} \mathrm{and} \mathrm{the}$ results are shown in Table 2. While BSA $0.25 \%$ did not increase the MIC for POA or PZA, the PZA and POA MIC was increased in presence of BSA $0.5-2 \%$, with higher BSA concentrations causing more increase in POA and PZA MIC. The influence of BSA on PZA or POA MIC was more pronounced for POA than for PZA at BSA $0.5,1$ and $2 \%$.

To determine how BSA might increase the MIC of POA and PZA, the study first tested the effect of varying concentrations of BSA on the medium $\mathrm{pH}$ in Sauton's liquid medium (Fig. 2a). BSA slightly decreased the $\mathrm{pH}$ of the mildly alkaline medium ( $\mathrm{pH}$ 7.5)

Table 2. Effect of BSA on susceptibility to POA and PZA

\begin{tabular}{lcc}
\hline $\begin{array}{l}\text { BSA concentration } \\
(\%)\end{array}$ & ${\text { POA } \mathrm{MIC}^{*}}^{*}$ & $\mathrm{PZA} \mathrm{MIC}^{*}$ \\
\hline 0 & 60 & 60 \\
0.25 & 70 & 70 \\
0.5 & 110 & 80 \\
1 & 150 & 80 \\
2 & 240 & 160 \\
\hline
\end{tabular}

* MIC here is $50 \%$ inhibition over control and expressed in $\mathrm{mg} / \mathrm{L}$. Details of the experiment are described in Materials and methods. towards neutral $\mathrm{pH}$. It is worth noting that BSA increased the $\mathrm{pH}$ of acidic medium ( $\mathrm{pH} 5.6$ ) by $c .1 \mathrm{pH}$ unit at $5-10 \%$, especially at high concentrations. Similarly, fetal bovine serum (FBS), which contains BSA, was also found to increase the medium $\mathrm{pH}$ (Fig. 2b). For example, $10 \%$ FBS was found to increase the medium $\mathrm{pH}$ from 5.5 to 6.5 , which is expected to increase the MIC by 10 -fold (Table 1). Because POA susceptibility was more affected than PZA even in presence of the same concentration of BSA, it was suspected that BSA might bind POA anion as a possible cause for the preferential decrease of susceptibility to POA over PZA. To test this, $\left[{ }^{14} \mathrm{C}\right] \mathrm{POA}$ and $\left[{ }^{14 C}\right]$ PZA were incubated with varying concentrations of BSA and then the mixture was passed through a membrane with a $30-\mathrm{kDa}$ mol. wt cut-off point, which will trap BSA with a mol. wt of $66 \mathrm{kDa}$. BSA was found to bind to POA significantly but not to PZA at $0.4,2$ and $10 \%$ (Fig. 3). The above results suggest that BSA $0.5-10 \%$ can antagonise the activity of POA by a combined action of neutralising the medium $\mathrm{pH}$ and binding to POA.

\section{Effect of bacterial growth phase on susceptibility to $P Z A$}

While it is clear that PZA is active against semidormant tubercle bacilli at acid $\mathrm{pH}$ [1], it is not known if the bacilli at different growth phase or physiological state would have different susceptibility to PZA. To address this, the study tested survival of a 4-day-old log phase culture and a 3-month-old stationary phase culture upon exposure to PZA for 3 days in an acidic phosphate-citrate buffer ( $\mathrm{pH}$ 5.6). The log phase and the 3-month-old cultures were concentrated to the same cell density and gave similar cfu counts. After the PZA exposure, cells from both 4-day-old culture and 3month-old culture were plated on $7 \mathrm{H} 11$ agar plates without PZA to assess the killing by PZA. Interestingly, the log phase cells were four-fold less susceptible to PZA exposure than the 3-month-old culture. PZA killed a comparable number of $\log$ phase bacilli at $250 \mathrm{mg} / \mathrm{L}$, whereas PZA killed a comparable number of stationary phase bacilli at $62 \mathrm{mg} / \mathrm{L}$. However, there 

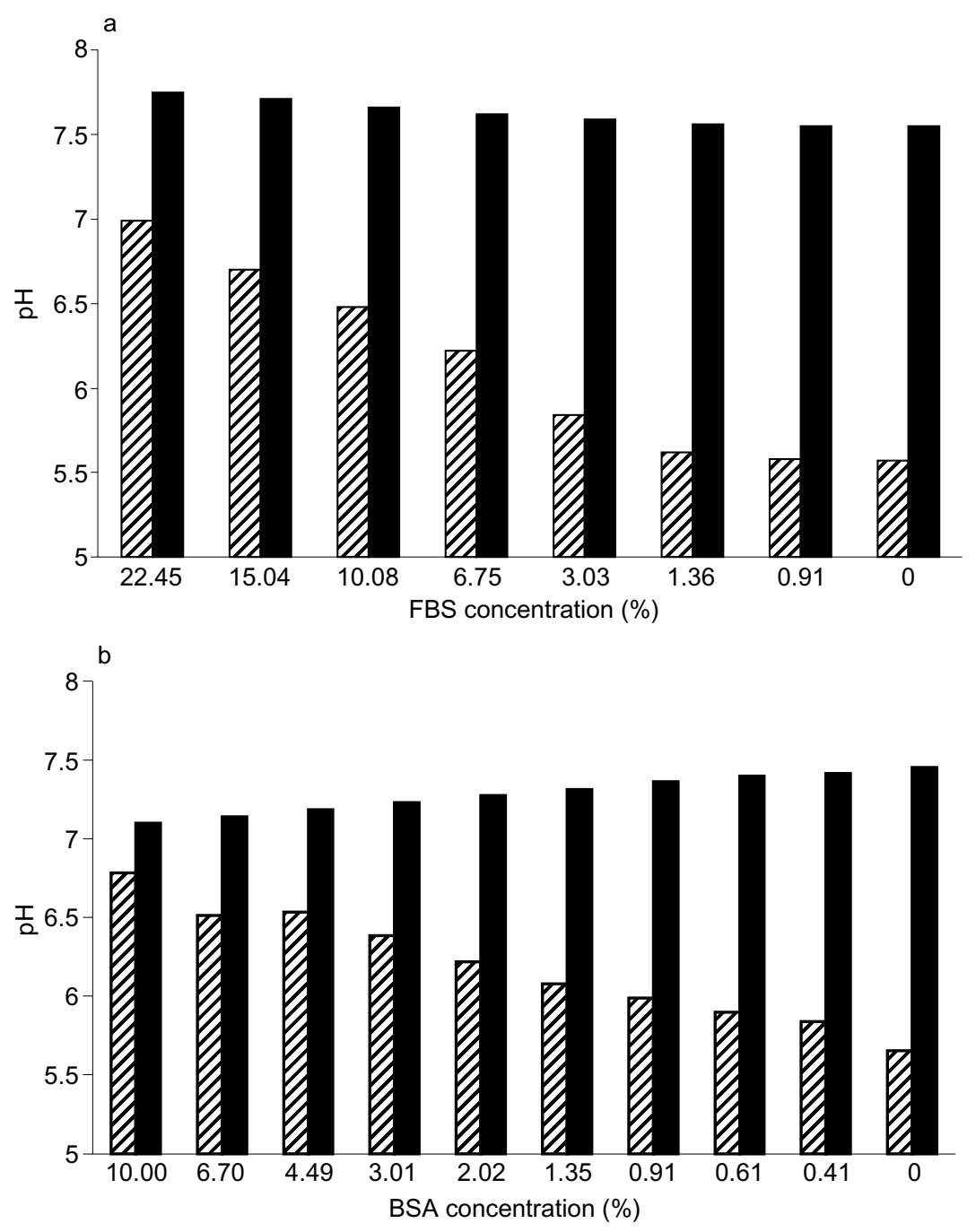

Fig. 2. Effect of BSA and FBS on medium pH. Varying concentrations of BSA or FBS were added to Sauton's medium preadjusted to $5.6(\varangle)$ and $7.5(\mathbf{\square})$. After incubation at room temperature for $1 \mathrm{~h}$, the $\mathrm{pH}$ of the medium was measured.

was no apparent difference in PZA MIC when the two cultures (without prior PZA exposure) were used directly for PZA susceptibility testing on 7H11 agar plates containing PZA. In a separate experiment involving two different cultures of 2 weeks old and 2.5 months old, the same finding was observed, i.e., old stationary phase cultures were more susceptible to PZA exposure than young cultures.

\section{Effect of reserpine and valinomycin on PZA or POA MIC}

It was shown in a previous study that reserpine, a multi-drug-resistant (MDR) efflux pump inhibitor [28], could cause increased accumulation of POA in $M$. tuberculosis [15]. Valinomycin, which disrupts membrane potential, was found to cause somewhat less POA accumulation in $M$. tuberculosis than reserpine [15]. This raised the question as to whether increased accumulation of POA caused by the efflux pump inhibitors might affect the susceptibility of $M$. tuberculosis to PZA or POA. Therefore, the susceptibility of M. tuberculosis H37Ra to PZA and POA in the presence of sublethal concentrations of reserpine and valinomycin was tested at $\mathrm{pH} 5.6$ and $\mathrm{pH} 6.8$. While valinomycin had no apparent effect on the susceptibility of H37Ra to PZA or POA (data not shown), reserpine, which blocks the POA efflux activity, was found to enhance the susceptibility to PZA but not to $\mathrm{POA}$ at both neutral $\mathrm{pH}$ and acid $\mathrm{pH}$. The enhancement of PZA susceptibility by reserpine was more apparent at $\mathrm{pH} 6.8$, with an MIC of $c .400 \mathrm{mg} / \mathrm{L}$ at reserpine concentrations of $20-40 \mu \mathrm{M}$. In the absence of reserpine, strain $\mathrm{H} 37 \mathrm{Ra}$ was resistant to PZA $\leqslant 1000 \mathrm{mg} / \mathrm{L}$ at $\mathrm{pH} 6.8$.

\section{Discussion}

Despite the importance of PZA in the current shortcourse TB chemotherapy, unlike other front-line TB drugs isoniazid, rifampin and ethambutol, PZA susceptibility testing is not performed in many clinical microbiology laboratories because of significant difficulty with PZA susceptibility testing. This difficulty mainly results from the acid $\mathrm{pH}$ requirement of PZA for drug activity. A most common problem is false resistance, i.e., a susceptible strain can behave like a resistant 


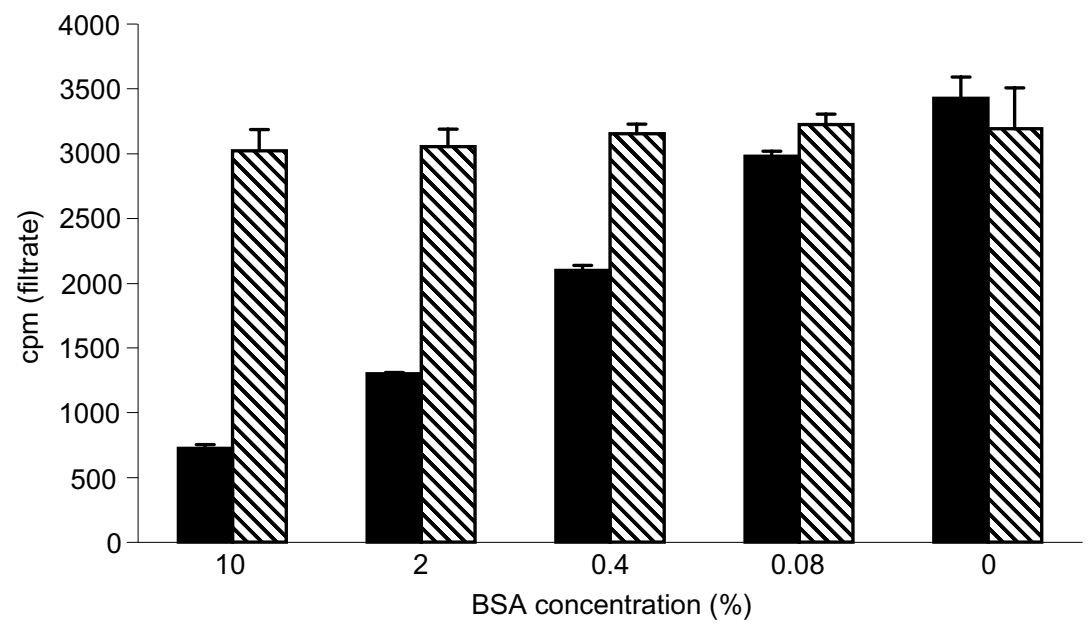

Fig. 3. Binding of POA by BSA. BSA at varying concentrations was incubated with $\left[{ }^{14} \mathrm{C}\right] \mathrm{POA}$ or $\left[{ }^{14} \mathrm{C}\right] \mathrm{PZA}$ in triplicate. BSA was found to significantly bind POA $(\square)$ but not PZA $(\mathbb{})$ as shown by a decrease in the radioactive counts in the filtrate.

strain if too high an inoculum is used [12]. For example, McDermott and colleagues showed that a 10fold increase in inoculum size $\left(10^{7} \mathrm{bacilli} / \mathrm{ml}\right)$ for a susceptible strain caused a significant increase in MIC of PZA (false resistance) [12]. Indeed, the present study has shown that large inocula $\left(10^{7-8}\right.$ cells $\left./ \mathrm{ml}\right)$ increased the medium $\mathrm{pH}$ from acidic $\mathrm{pH}$ towards neutral $\mathrm{pH}$, a finding consistent with a previous study [21]. On the other hand, an inoculum size of $\leqslant 10^{6}$ cells $/ \mathrm{ml}$ did not have significant effect on medium $\mathrm{pH}$ (Fig. 1a). This finding is consistent with the inoculum size $\left(\leqslant 10^{6}\right.$ cells $\left./ \mathrm{ml}\right)$ commonly used for PZA susceptibility testing that does not lead to false resistance [12]. The rate of PZA killing is a function of acidity of medium $\mathrm{pH}[1,14]$, which is shown to increase with high bacterial inoculum (Fig. 1). This has particular relevance to PZA, which kills tubercle bacilli slowly and incompletely [1]. The increase in medium $\mathrm{pH}$ over time by high inoculum would relieve acid inhibition and allow bacilli to grow even in presence of PZA, which will cause significant increase of PZA MIC. This increase of medium $\mathrm{pH}$ by high inoculum depends on live bacilli, and heat-killed bacilli hardly affected the $\mathrm{pH}$ of the medium (Fig. 1). This is consistent with the observation that live bacterial cells neutralise the medium $\mathrm{pH}$ when subjected to acidic $\mathrm{pH}$ by de-amination and decarboxylation of certain amino acids [29]. Such a mechanism must also be operative in the tubercle bacillus as judged by neutralisation of medium $\mathrm{pH}$ by a large inoculum as seen in this study.

There have been inconsistent reports regarding POA MIC for M. tuberculosis. Heifets and colleagues reported higher POA MIC $(240-480 \mathrm{mg} / \mathrm{L})$ than PZA MIC $(16-50 \mathrm{mg} / \mathrm{L})$ in liquid $7 \mathrm{H} 12$ medium [22], whereas Cynamon and colleagues reported comparable POA and PZA MIC in liquid Dubos medium [23]. Both $7 \mathrm{H} 12$ and Dubos liquid media contained BSA $0.5 \%$ and the only significant difference is the solvent used to dissolve POA. In the study that reported higher POA MIC, hot water was used to dissolve POA [22], whereas in the study that reported comparable POA and PZA MIC, DMSO was used to dissolve POA [23]. The higher POA MIC than PZA MIC reported previously probably reflects the poor solubility of POA in water, such that the actual POA in the stock solution and medium is less than intended due to insoluble POA precipitates. The present study has shown that $M$. tuberculosis was equally susceptible to both PZA and POA on 7H11 agar medium when POA was dissolved in DMSO or made into a POA salt. Another cause for the higher POA MIC than PZA MIC is BSA concentration, where at $>0.5 \%$ (i.e., at $1 \%$ and $2 \%$ ) it preferentially increased the POA MIC over PZA MIC (Table 2). This can be explained by the finding that BSA binds to weak acid POA but not neutral amide PZA, as shown in Fig. 3. This finding is consistent with the well-known observation that BSA can bind a range of compounds including weak acids. The effect of BSA on antagonising POA activity can be shown in medium with and without BSA. In Sauton's agar medium without BSA, the PZA or POA MIC (25$32 \mathrm{mg} / \mathrm{L}$ ) was two-fold lower than when supplemented with BSA $0.5 \%$ or $7 \mathrm{H} 11$ agar containing BSA $0.5 \%$ as part of the ADC supplement. It was found that the effect of BSA on increasing the MIC of PZA can be caused by neutralisation of acid medium (Fig. 2) and binding of POA by BSA (Fig. 3), although the relative contribution of the two factors to POA and PZA MIC may vary according to the medium (liquid versus solid medium) used, BSA concentration and $\mathrm{pH}$. Heifets and colleagues developed a new agar-based medium at pH 6.0 for PZA susceptibility testing [30], which contains fetal bovine serum $10 \%$. The FBS $10 \%$ is a significant source of BSA and indeed, the PZA MIC of the Heifets medium is very high, with a cut-off value of $900-1200 \mathrm{mg} / \mathrm{L}$ [30]. This MIC (900-1200 mg/L) is c. 5-6-fold higher than the MIC $(200 \mathrm{mg} / \mathrm{L})$ in 7H12B medium containing BSA 0.5\% [14] (Table 1). This higher than predicted PZA MIC could be explained by the BSA effects observed in the present study.

An earlier study showed that M. tuberculosis has a 
much weaker efflux mechanism for POA than $M$. smegmatis and this difference in POA efflux activity relates to the susceptibility or resistance to PZA [15]. The weak efflux mechanism in M. tuberculosis can be inhibited by the multi-drug resistance efflux pump inhibitor reserpine, and less so by valinomycin, an agent that disrupts membrane potential [15]. The present study showed that reserpine enhanced the susceptibility of H37Ra to PZA from $>1000$ to $400 \mathrm{mg} / \mathrm{L}$ even near neutral $\mathrm{pH}$ 6.8. The fact that the PZA MIC is lowered in the presence of reserpine indicates that POA accumulation is toxic to $M$. tuberculosis even at neutral $\mathrm{pH}$. In addition, the fact that the PZA MIC in presence of reserpine is not as low as at acid $\mathrm{pH}$ suggests that POA anion accumulation itself has only limited effect on killing of M. tuberculosis, and that acid $\mathrm{pH}$ plays an important role in PZA action, possibly by recycling of POA, leading to de-energised membrane and bacterial death [17]. While reserpine enhanced susceptibility to PZA, it had no apparent effect on POA MIC. This can be explained by the differential transport of PZA over POA across the membrane. POA at near neutral $\mathrm{pH} 6.8$ is $99.9 \%$ charged (Table 1) and cannot penetrate bacterial cells easily, therefore, reserpine had no effect on POA MIC. In contrast, PZA as a neutral amide gets into cells readily and is converted intracellularly to POA, which is subject to the POA efflux inhibitory activity of reserpine [15]. On the other hand, valinomycin did not have any significant effect on PZA or POA susceptibility. This finding is in keeping with the degree of POA accumulation caused by reserpine versus valinomycin, where reserpine caused higher POA accumulation in $M$. tuberculosis than valinomycin [15].

In conclusion, PZA susceptibility testing is difficult and is subject to various problems such as false resistance. The findings reported in this study provide useful explanations for the inconsistent results of PZA and POA susceptibility testing [18], the relationship between pH and PZA MIC [14], the effect of BSA on PZA susceptibility testing [30] and the discrepant POA susceptibility $[22,23]$. These findings may have implications for improved PZA susceptibility testing in clinical microbiology laboratories.

This study was supported by NIH grants (AI40584 and AI44063) and the Potts Memorial Foundation. We thank NIH AIDS Reagents Program for the provision of $\left[{ }^{14} \mathrm{C}\right] \mathrm{PZA}$.

\section{References}

1. Heifets L, Lindholm-Levy P. Pyrazinamide sterilizing activity in vitro against semidormant Mycobacterium tuberculosis bacterial populations. Am Rev Respir Dis 1992; 145: 1223-1225.

2. Konno K, Feldmann FM, McDermott W. Pyrazinamide susceptibility and amidase activity of tubercle bacilli. Am Rev Respir Dis 1967; 95: 461-469.

3. Scorpio A, Zhang Y. Mutations in $p n c A$, a gene encoding pyrazinamidase/nicotinamidase, cause resistance to the antituberculous drug pyrazinamide in tubercle bacillus. Nature Med 1996; 2: 662-667.
4. Scorpio A, Lindholm-Levy P, Heifets L et al. Characterization of pncA mutations in pyrazinamide-resistant Mycobacterium tuberculosis. Antimicrob Agents Chemother 1997; 41: $540-543$.

5. Sreevatsan S, Pan X, Zhang Y, Kreiswirth BN, Musser JM. Mutations associated with pyrazinamide resistance in $p n c A$ of Mycobacterium tuberculosis complex organisms. Antimicrob Agents Chemother 1997; 41: 636-640.

6. Hirano K, Takahashi M, Kazumi Y, Fukasawa Y, Abe C. Mutation in pncA is a major mechanism of pyrazinamide resistance in Mycobacterium tuberculosis. Tuberc Lung Dis 1997; 78: 117-122.

7. Lemaitre N, Sougakoff W, Truffot-Pernot C, Jarlier V. Characterization of new mutations in pyrazinamide-resistant strains of Mycobacterium tuberculosis and identification of conserved regions important for the catalytic activity of the pyrazinamidase PncA. Antimicrob Agents Chemother 1999; 43: 1761-1763.

8. Marttila HJ, Marjamaki M, Vyshnevskaya E et al. pncA mutations in pyrazinamide-resistant Mycobacterium tuberculosis isolates from northwestern Russia. Antimicrob Agents Chemother 1999; 43: 1764-1766.

9. Mestdagh M, Fonteyne PA, Realini L et al. Relationship between pyrazinamide resistance, loss of pyrazinamidase activity, and mutations in the $p n c A$ locus in multidrug-resistant clinical isolates of Mycobacterium tuberculosis. Antimicrob Agents Chemother 1999; 43: 2317-2319.

10. Cheng S-J, Thibert L, Sanchez T, Heifets L, Zhang Y. pncA mutations as a major mechanism of pyrazinamide resistance in Mycobacterium tuberculosis: spread of a monoresistant strain in Quebec, Canada. Antimicrob Agents Chemother 2000; 44: 528-532.

11. Morlock GP, Crawford JT, Butler WR et al. Phenotypic characterization of pncA mutants of Mycobacterium tuberculosis. Antimicrob Agents Chemother 2000; 44: 2291-2295.

12. McDermott W, Tompsett R. Activation of pyrazinamide and nicotinamide in acidic environment in vitro. Am Rev Tuberc 1954; 70: 748-754.

13. Tarshis MS, Weed WA. Lack of significant in vitro sensitivity of Mycobacterium tuberculosis to pyrazinamide on three different solid media. Am Rev Tuberc 1953; 67: 391-395.

14. Salfinger M, Heifets LB. Determination of pyrazinamide MICs for Mycobacterium tuberculosis at different $\mathrm{pH}$ by the radiometric method. Antimicrob Agents Chemother 1988; 32: $1002-1004$.

15. Zhang Y, Scorpio A, Nikaido H, Sun Z. Role of acid $\mathrm{pH}$ and deficient efflux of pyrazinoic acid in unique susceptibility of Mycobacterium tuberculosis to pyrazinamide. J Bacteriol 1999; 181: $2044-2049$.

16. Zimhony O, Cox JS, Welch JT, Vilchèze C, Jacobs WR. Pyrazinamide inhibits the eukaryotic-like fatty acid synthetase I (FAS-I) of Mycobacterium tuberculosis. Nature Med 2000; 6: 1043-1047.

17. Zhang Y, Telenti A. Genetics of drug resistance in Mycobacterium tuberculosis. In: Jacobs WR, Hatfull GF (eds) Molecular genetics of mycobacteria. Washington, DC, ASM Press. 2000: 235-254.

18. Hewlett D, Horn DL, Alfalla C. Drug-resistant tuberculosis: inconsistent results of pyrazinamide susceptibility testing. JAMA 1995; 273: 916-917.

19. Siddiqi SH. Antimicrobial susceptibility testing: radiometric (BACTEC) tests for slowly growing mycobacteria. In: Isenberg HD (ed) Clinical microbiology procedure handbook. Washington, DC, ASM Press. 1992: 14-25.

20. Bonicke R, Lisboa BP. Typendifferenzierung der Tuberkulosebakterien mit Hilfe des Nikotinamidasetests. Tuberkulosearzt 1959; 13: 375-377.

21. Dickinson JM, Mitchison DA. Observations in vitro on the suitability of pyrazinamide for intermittent chemotherapy of tuberculosis. Tubercle 1970; 51: 389-396.

22. Heifets LB, Flory MA, Lindholm-Levy PJ. Does pyrazinoic acid as an active moiety of pyrazinamide have specific activity against Mycobacterium tuberculosis? Antimicrob Agents Chemother 1989; 33: 1252-2154.

23. Speirs RJ, Welch JT, Cynamon MH. Activity of $n$-propyl pyrazinoate against pyrazinamide-resistant Mycobacterium tuberculosis: investigations into mechanism of action of and mechanism of resistance to pyrazinamide. Antimicrob Agents Chemother 1995; 39: 1269-1271. 
24. Allen BW. Mycobacteria: general culture methodology and safety considerations. In: Parish T, Stoker NG (eds) Mycobacteria protocols. Methods in molecular biology, vol 101. Totowa, NJ, Humana Press. 1998: 15-30.

25. Stottmeier KD, Beam RE, Kubica GP. Determination of drug susceptibility of mycobacteria to pyrazinamide in 7H10 agar. Am Rev Respir Dis 1967; 96: 1072-1075.

26. Sun Z, Zhang Y. Antituberculosis activity of certain antifungal and antihelmintic drugs. Tuberc Lung Dis 1999; 79: 319-320.

27. Budavari S (ed). The Merck Index. An encyclopedia of chemicals, drugs, and biologicals. Rahway, NJ, Merck. 11th edn. 1989.

28. Neyfakh AA, Bidnenko VE, Chen LB. Efflux-mediated multidrug resistance in Bacillus subtilis: similarities and dissimilarities with the mammalian system. Proc Natl Acad Sci USA 1991; 88: 4781-4785.

29. Moat AG, Foster JW. Growth and its regulation. In: Microbial physiology, 3rd edn. New York, Wiley-Liss. 1995: 518-548.

30. Heifets L, Sanchez T. New agar medium for testing susceptibility of Mycobacterium tuberculosis to pyrazinamide. $J$ Clin Microbiol 2000; 38: 1498-1501. 